\title{
Efficiency of heat engines coupled to nonequilibrium reservoirs
}

\author{
Obinna $\mathrm{Abah}^{1,2}$ and Eric $\mathrm{Lutz}^{2,3}$ \\ ${ }^{1}$ Department of Physics, University of Augsburg, D-86135 Augsburg, Germany \\ ${ }^{2}$ Dahlem Center for Complex Quantum Systems, FU Berlin, D-14195 Berlin, Germany \\ ${ }^{3}$ Institute for Theoretical Physics, University of Erlangen-Nürnberg, D-91058 Erlangen, Germany
}

\begin{abstract}
We consider quantum heat engines that operate between nonequilibrium stationary reservoirs. We evaluate their maximum efficiency from the positivity of the entropy production and show that it can be expressed in terms of an effective temperature that depends on the nature of the reservoirs. We further compute the efficiency at maximum power for different kinds of engineered reservoirs and derive a nonequilibrium generalization of the Clausius statement of the second law.
\end{abstract}

PACS numbers: 05.30.-d, 03.65.-w

Engines are devices that convert various forms of energy into useful mechanical work and motion. In thermodynamics, two different kinds of machines can be distinguished. On the one hand, there are heat engines that operate between two reservoirs at different temperatures, such as internal combustion engines [1, 2]. On the other hand, there are molecular motors that are driven from equilibrium by varying external parameters, while in contact with a single isothermal reservoir [3, 4]. The latter describe biological motor proteins as well as artificial nanomachines 5, 6. An essential characteristic of any machine is its efficiency defined as the ratio of work output to energy input. Whereas for heat engines the efficiency is limited by the Carnot formula, $\eta_{c}=1-T_{1} / T_{2}$, where $T_{1}$ and $T_{2}$ are the temperatures of the two thermal reservoirs $\left(T_{1}<T_{2}\right)$, it can reach unity for molecular motors 7-10. Maximum efficiency usually corresponds to quasistatic conditions, and therefore to zero power. A practically more relevant quantity is thus the efficiency at maximum power which for heat engines is given by $\eta_{c} / 2$ for small temperature differences 11 13. For molecular motors, the efficiency at maximum power can reach the thermodynamic limit 1 for strong driving [14, 15].

Heat engines are usually assumed to be in contact with two equilibrium reservoirs. In this paper, we investigate the more general case where the engine runs between stationary nonequilibrium reservoirs. In a sense, this situation interpolates between traditional heat engines and molecular motors. Indeed, the efficiency of these heat engines may be larger than the Carnot efficiency and they may operate isothermally. Our study is motivated by the recent advent of reservoir engineering techniques in quantum optical systems, such as ion traps [16, 17, microwave cavities [18, 19, optical lattices [20, 21] and optomechanical systems 22, that enable the preparation of nonthermal environments. In addition, theoretical studies have shown in individual cases that the efficiency of heat engines coupled to nonthermal quantum coherent 23] or quantum correlated 24] reservoirs may sometimes exceed the Carnot value. The two fundamental questions that we here address are therefore: i) what is the maximum (universal) efficiency that may be reached, and ii) under what conditions is this efficiency larger than the standard Carnot limit? In the following, we consider a quantum heat engine coupled to general stationary nonthermal reservoirs. It will be convenient to regard these reservoirs as perturbed thermal reservoirs. They will then be characterized by a temperature and a second parameter (or more) that quantifies the deviation from equilibrium, such as the degree of quantum coherence 23 or the amount of quantum correlations 24 . We begin by performing a detailed analysis of the quantum Otto cycle for a time-dependent harmonic oscillator, a paradigm of quantum heat engines and a generalization of the common four-stroke car engine [25 29]. We evaluate its efficiency which we express in terms of the Hamiltonian of mean force [30, 31, a quantum extension of the potential of mean force known in the statistical theory of fluids 32. We derive an explicit expression for the maximum efficiency of a heat engine from the condition of positive entropy production of the second law of thermodynamics 33. This efficiency may be larger or smaller than the Carnot efficiency depending on the properties of the reservoirs, which we quantify with an effective temperature. We further obtain a generalization of the Clausius statement of the second law on the direction of heat flow between nonequilibrium systems characterized by their effective temperatures. Finally, we compute the efficiency at maximum power of the Otto engine for the concrete examples of correlated and coherent quantum reservoirs.

Quantum Otto engine. We consider a quantum Otto engine whose working medium is a harmonic oscillator with time-dependent frequency $\omega_{t}$ [25]29]. The Otto cycle consists of two isentropic processes during which the frequency is unitarily varied between $\omega_{1}$ and $\omega_{2}$, and of two isochoric (constant frequency) processes during which the oscillator is connected to two different reservoirs (see Fig. 1). A concrete scheme to experimentally realize such an engine using a single ion in a linear Paul trap has been proposed in Ref. 29]. In the usual Otto cycle, the two reservoirs are assumed to be thermal and characterized by the inverse temperatures $\beta_{i}=1 /\left(k T_{i}\right)$, $(i=1,2)$, where $k$ is the Boltzmann constant. Here, we examine the situation where the engine is alternatingly 


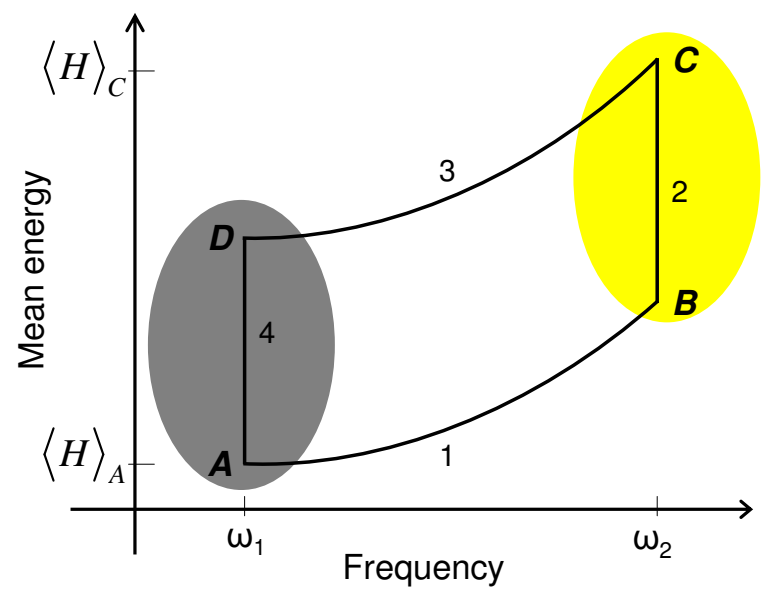

FIG. 1: (Color online) Energy-frequency diagram of the quantum Otto engine. The thermodynamic cycle consists of two isentropic (stroke 1 and 3) and two isochoric (stroke 2 and 3 ) processes. In the latter, the heat engine is coupled to engineered nonthermal stationary reservoirs that are described by a temperature and additional parameters that characterize the deviation from thermal equilibrium.

coupled to engineered nonthermal reservoirs. For simplicity, we will first focus on the case where only the hot reservoir is nonthermal. When connected to this reservoir, the oscillator relaxes to a nonequilibrium state that we write in the general form, $\rho_{2}=\exp \left[-\beta_{2}\left(H_{2}+\Delta H_{2}\right)\right] / Z_{2}^{*}$, where $H_{2}=p^{2} /(2 m)+m \omega_{2}^{2} x^{2} / 2$ is the Hamiltonian of the oscillator at frequency $\omega_{2}$ ( $m$ denotes the mass) and $Z_{2}^{*}$ the normalization constant. The operator $\Delta H_{2}$ quantifies the departure from equilibrium and may be arbitrary [34, 35. We note that the operator $H_{2}^{*}=H_{2}+\Delta H_{2}$ can be seen as a Hamiltonian of mean force [30, 31]. It is important to stress that the state $\rho_{2}$ should be centered, $\langle x\rangle=\langle p\rangle=0$, so that the nonequilibrium reservoir is a proper heat source that can only exchange heat with the engine, but no work [36]. The mean energy of the harmonic oscillator in the nonequilibrium state is then,

$$
\left\langle H_{2}\right\rangle=\hbar \omega_{2}\left(\bar{n}_{2}+\Delta \bar{n}+1 / 2\right),
$$

where $\bar{n}_{i}=\left[\exp \left(\hbar \beta_{i} \omega_{i}\right)-1\right]^{-1}$ is the mean occupation number of a thermal quantum oscillator and $\Delta \bar{n}=$ $\Delta \bar{n}\left(\omega_{2}\right)$ that associated with the deviation from the thermal state. The density operator of the harmonic oscillator in contact with the cold thermal reservoir is $\rho_{1}=$ $\exp \left(-\beta_{1} H_{1}\right) / Z_{1}$ with mean energy $\left\langle H_{1}\right\rangle=\hbar \omega_{1}\left(\bar{n}_{1}+1 / 2\right)$.

During the two isentropic parts of the thermodynamic cycle (stroke 1 and 3), the time-dependent oscillator is isolated and its dynamics is hence unitary. As a result, its Schrödinger equation can be solved exactly using a Gaussian wave function ansatz and the mean energy can be evaluated analytically [37-39]. During the two isochoric branches (stroke 2 and 4), the oscillator relaxes respec- tively to a nonequilibrium and an equilibrium state. The corresponding average energies of the harmonic oscillator at the four corners of the quantum Otto cycle are,

$$
\begin{aligned}
\langle H\rangle_{A} & =\hbar \omega_{1}\left(\bar{n}_{1}+1 / 2\right), \\
\langle H\rangle_{B} & =\hbar \omega_{2} Q_{1}^{*}\left(\bar{n}_{1}+1 / 2\right), \\
\langle H\rangle_{C} & =\hbar \omega_{2}\left(\bar{n}_{2}+\Delta \bar{n}+1 / 2\right), \\
\langle H\rangle_{D} & =\hbar \omega_{1} Q_{2}^{*}\left(\bar{n}_{2}+\Delta \bar{n}+1 / 2\right),
\end{aligned}
$$

where we have used Eq. (1) and Eq. (5.12) of Ref. 37. We note that the energies at point $C$ and $D$ are modified by the presence of the nonthermal reservoir. The two parameters $Q_{1}^{*}$ and $Q_{2}^{*}$ characterize the degree of adiabaticity of the compression and expansion phases 1 and 3 37. Their explicit expressions for any given frequency modulation $\omega_{t}$, can be found in Refs. [38, 39. They are, for example, equal to one for adiabatic processes and to $\left(\omega_{1}^{2}+\omega_{2}^{2}\right) /\left(2 \omega_{1} \omega_{2}\right)$ for a sudden frequency change.

To evaluate the efficiency of the Otto engine, we need to compute work and heat along the four branches of the cycle (see Fig. 1). The mean works, denoted by $\left\langle W_{1}\right\rangle$ and $\left\langle W_{3}\right\rangle$, done during stroke 1 and 3 are given by,

$$
\begin{aligned}
& \left\langle W_{1}\right\rangle=\langle H\rangle_{B}-\langle H\rangle_{A}=\left(\hbar \omega_{2} Q_{1}^{*}-\hbar \omega_{1}\right)\left(\bar{n}_{1}+\frac{1}{2}\right), \\
& \left\langle W_{3}\right\rangle=\langle H\rangle_{D}-\langle H\rangle_{C}=\left(\hbar \omega_{1} Q_{2}^{*}-\hbar \omega_{2}\right)\left(\bar{n}_{2}+\Delta \bar{n}+\frac{1}{2}\right) .
\end{aligned}
$$

At the same time, the mean heats, $\left\langle Q_{2}\right\rangle$ and $\left\langle Q_{4}\right\rangle$, exchanged with the reservoirs during stroke 2 and 4 read,

$$
\begin{aligned}
\left\langle Q_{2}\right\rangle & =\langle H\rangle_{C}-\langle H\rangle_{B} \\
& =\hbar \omega_{2}\left(\bar{n}_{2}+\Delta \bar{n}+\frac{1}{2}\right)-\hbar \omega_{2} Q_{1}^{*}\left(\bar{n}_{1}+\frac{1}{2}\right), \\
\left\langle Q_{4}\right\rangle & =\langle H\rangle_{A}-\langle H\rangle_{D} \\
& =\hbar \omega_{1}\left(\bar{n}_{1}+\frac{1}{2}\right)-\hbar \omega_{1} Q_{2}^{*}\left(\bar{n}_{2}+\Delta \bar{n}+\frac{1}{2}\right) .
\end{aligned}
$$

The efficiency, defined as the ratio of the total work per cycle, $\langle W\rangle=-\left(\left\langle W_{1}\right\rangle+\left\langle W_{3}\right\rangle\right)$, to the heat received from the hot reservoir, $\left\langle Q_{2}\right\rangle$, can then be written as,

$$
\eta=1-\frac{\omega_{1}}{\omega_{2}} \frac{\left(\bar{n}_{1}+1 / 2\right)-Q_{2}^{*}\left(\bar{n}_{2}+\Delta \bar{n}+1 / 2\right)}{Q_{1}^{*}\left(\bar{n}_{1}+1 / 2\right)-\left(\bar{n}_{2}+\Delta \bar{n}+1 / 2\right)} .
$$

The above quantum expression is exact. It gives the finite time efficiency of the quantum Otto engine for any frequency modulation $\omega_{t}$, any inverse temperature $\beta_{i}$, and any nonequilibrium stationary reservoir.

Maximum efficiency. The maximum efficiency can be evaluated from the positivity of the entropy production [1, 40]. Applying the Klein inequality, $S\left(\rho_{r} \| \rho_{s}\right) \geq 0$ [41], to the isochoric processes $B C$ and $D A$, we obtain,

$$
\begin{aligned}
& S\left(\rho_{B} \| \rho_{C}\right)+S\left(\rho_{D} \| \rho_{A}\right) \\
& =-\beta_{2}\left\langle Q_{2}\right\rangle-\beta_{1}\left\langle Q_{4}\right\rangle+\beta_{2} \operatorname{tr}\left\{\left(\rho_{B}-\rho_{C}\right) \Delta H_{2}\right\} \geq 0,(6)
\end{aligned}
$$

where $\rho_{r}$ is the density operator of state $r$ and the quantum relative entropy, $S\left(\rho_{r} \| \rho_{s}\right)=\operatorname{tr}\left\{\rho_{r}\left(\ln \rho_{r}-\ln \rho_{s}\right)\right\}$, 
the entropy production associated with the thermalization step $r \rightarrow s$ [42]. We note that the von Neumann entropy remains constant during the isentropic processes $A B$ and $C D$. To evaluate the last term in Eq. (6), we use that $\langle\Delta H\rangle_{r}=\operatorname{tr}\left\{\rho_{r} \Delta H_{2}\right\}=(a / 2)\left\langle p^{2}\right\rangle_{r}+(b / 2)\left\langle x^{2}\right\rangle_{r}$, where the two coefficients $a$ and $b$ are given by [31,

$$
a=\frac{1}{\beta_{2}\left\langle p^{2}\right\rangle_{C}}-\frac{1}{m}, \quad b=\frac{1}{\beta_{2}\left\langle x^{2}\right\rangle_{C}}-m \omega_{2}^{2},
$$

in the high-temperature limit, $\bar{n}_{i}+1 / 2 \simeq 1 /\left(\hbar \beta_{i} \omega_{i}\right)$. For small deviations from equilibrium, momentum and position quadratures at point $C$ can be written as $\left\langle p^{2}\right\rangle_{C} \simeq$ $m \hbar \omega_{2}\left(\bar{n}_{2}+\Delta \bar{n}\right)$ and $\left\langle x^{2}\right\rangle_{C} \simeq\left(\hbar / m \omega_{2}\right)\left(\bar{n}_{2}+\Delta \bar{n}\right)$. As a result, for adiabatic frequency modulation, $Q_{1,2}^{*}=1$, which corresponds to maximum efficiency,

$$
\begin{aligned}
\langle\Delta H\rangle_{B} & =k T_{1} \frac{\omega_{2}}{\omega_{1}}\left(\frac{T_{2}}{T_{2}^{\text {eff }}}-1\right), \\
\langle\Delta H\rangle_{C} & =k\left(T_{2}-T_{2}^{\text {eff }}\right) .
\end{aligned}
$$

In the above equations, we have introduced the effective temperature, $T_{2}^{\mathrm{eff}}=T_{2}+\hbar \omega_{2} \Delta \bar{n} / k=T_{2}+\langle\Delta H\rangle_{C} / k$, that quantifies the departure from equilibrium of the hot nonthermal reservoir. For adiabatic frequency modulation and high temperature, Eq. (4) can be expressed in the form, $\left\langle Q_{2}\right\rangle=k T_{2}^{\text {eff }}-k T_{1} \omega_{2} / \omega_{1}$. Hence $-\beta_{2}\left\langle Q_{2}\right\rangle+$ $\beta_{2}\left(\langle\Delta H\rangle_{B}-\langle\Delta H\rangle_{C}\right)=-\beta_{2}^{\text {eff }}\left\langle Q_{2}\right\rangle$, and the second law of thermodynamics yields the inequality,

$$
-\beta_{2}^{\text {eff }}\left\langle Q_{2}\right\rangle-\beta_{1}\left\langle Q_{4}\right\rangle \geq 0 .
$$

On the other hand, according to the first law,

$$
\langle W\rangle=\left\langle Q_{2}\right\rangle+\left\langle Q_{4}\right\rangle .
$$

Combining Eqs. (9) and (10), we eventually find $\left\langle Q_{2}\right\rangle\left(\beta_{1}-\beta_{2}^{\text {eff }}\right) \geq \beta_{1}\langle W\rangle$. We can therefore conclude that the maximum efficiency of the engine is,

$$
\eta_{\max }=1-\frac{\beta_{2}^{\mathrm{eff}}}{\beta_{1}}=1-\frac{\beta_{2}}{\beta_{1}\left(1+\beta_{2}\langle\Delta H\rangle_{C}\right)} .
$$

We note that since the work produced by the engine is positive, the heat $\left\langle Q_{2}\right\rangle \geq 0$ only if $T_{2}^{\text {eff }} \geq T_{1}$, that is, heat flows from the high (effective) temperature to the low temperature reservoir. In particular, in the absence of the heat engine, $\langle W\rangle=0$, heat will flow from the system at high effective temperature to the system at low effective temperature 43. We have thus generalized the Clausius statement of the second law [44 to stationary systems that are not in thermal equilibrium, extending analogous equilibrium derivations found e.g. in Refs. [45, 46].

The Clausius principle provides alternative means to derive the maximum efficiency [36. Assuming that $T_{2}^{\mathrm{eff}} \geq T_{1}$, we observe that heat is absorbed from the hot reservoir, $\left\langle Q_{2}\right\rangle \geq 0$, and flows into the cold reservoir, $\left\langle Q_{4}\right\rangle \leq 0$. Equation (4) then leads to,

$$
\frac{\bar{n}_{2}+\Delta \bar{n}+1 / 2}{\bar{n}_{1}+1 / 2} \geq Q_{1}^{*}, \quad \frac{\bar{n}_{1}+1 / 2}{\bar{n}_{2}+\Delta \bar{n}+1 / 2} \leq Q_{2}^{*} .
$$

Combining Eqs. (5) and (12) for adiabatic frequency modulation, $Q_{1,2}^{*}=1$, and high temperatures, we recover the maximum efficiency (11).

Equation (11) generalizes the standard Carnot formula to heat engines that operate between a thermal and a nonthermal stationary reservoir. It only depends on the two temperatures of the reservoirs and on the energy deviation from equilibrium at point $C,\langle\Delta H\rangle_{C}=$ $\langle H\rangle_{C}-\langle H\rangle_{C}^{\mathrm{eq}}=\hbar \omega_{2} \Delta \bar{n}$. Expression 111 exceeds the Carnot efficiency $\eta_{c}$ when $\langle\Delta H\rangle_{C}>0$. This condition corresponds to a larger area of the thermodynamic cycle in Fig. 1, and therefore to a larger work output. Equation (11) further indicates that the nonthermal reservoir can be described by an effective temperature, $T_{2}^{\text {eff }}$, that may be larger or smaller than that of the unperturbed thermal reservoir (see below). This situation is reminiscent of that of molecular motors where the external driving is sometimes regarded as a nonequilibrium reservoir with an effective temperature (see e.g. Ref. [4, Sect. 3.4.2).

Expression (11) agrees with the maximum efficiency obtained for a quantum Carnot engine in contact with either a quantum coherent [23] or a quantum correlated 24] reservoir (see below). We have here generalized these results to quantum nonthermal reservoirs of arbitrary nature. We note, moreover, that the fact that Eq. (11) appears as the maximum efficiency of different kinds of quantum heat engines (Otto and particularly Carnot) strongly hints at its universal validity for all heat engines.

An important observation is that in thermodynamics the two thermal reservoirs are supposed to be given 1, 2 . In particular, the energetic cost of preparing, say, a high temperature reservoir in addition to an ambient low temperature reservoir is not taken into account in the calculation of the efficiency of a heat engine [47. Such an inclusion would indeed lead to vanishing efficiencies due to the large (strictly speaking infinite) energy content of a proper heat reservoir. We here follow the same approach and consider the nonthermal reservoir as given. In this framework, the Carnot formula appears as an expression of the second law of thermodynamics for a specific form of nonequilibrium (two thermal reservoirs at different temperatures), whereas the efficiency (11) applies to a more general form of nonequilibrium (one thermal and one nonthermal reservoir). In a similar manner, the maximum efficiency of 1 of molecular motors is a consequence of the second law for this yet different type of nonequilibrium [48. It is worth noticing that Eq. 111) yields a non-zero result, $\eta_{\max }=\beta_{2}\langle\Delta H\rangle_{C} /\left(1+\beta_{2}\langle\Delta H\rangle_{C}\right)$, for isothermal reservoirs, $\beta_{1}=\beta_{2}$. In this situation, which is akin to that of molecular motors, the nonthermal reservoir can be seen as an external nonequilibrium driving.

Efficiency at maximum power. The efficiency at maximum power is often a more relevant quantity than the maximum efficiency which corresponds to zero power [49. In contrast to the latter, however, there does not seem to be a universal expression for the efficiency at 
maximum power; it not only usually depends on the optimization procedure, but also, as we will show, on the details of the nonthermal reservoir. The power output of a heat engine is defined as $P=\langle W\rangle / \tau$, where $\tau$ is the duration of the cycle. In the following, we evaluate the maximum efficiency and the efficiency at maximum power for two different examples. For simplicity, we focus on adiabatic compression and expansion, $Q_{1,2}^{*}=1$, since nonadiabatic processes lead to smaller efficiencies [27, 29], and to the high-temperature regime $\beta_{i} \hbar \omega_{i} \ll 1$.

Let us consider a quantum photo-engine made of a single mode (the harmonic oscillator) in a resonant cavity with moving mirror, and coupled to a beam of thermal two-level atoms that pass through the cavity [23, 24]. When the atoms are uncorrelated, the beam plays the role of a thermal reservoir. By contrast, for correlated atoms the engineered reservoir is nonthermal. For pairwise thermally entangled atoms, the deviation of the mean occupation number from equilibrium is [24],

$$
\Delta \bar{n}_{\lambda: 1}=\frac{\beta_{2} \hbar \lambda^{2}}{4 \omega_{2}}, \quad \Delta \bar{n}_{\lambda: 2}=-\frac{\lambda}{2 \omega_{2}},
$$

when respectively one or the two atoms of a correlated pair fly through the cavity, in the limit of high temperature, $\beta_{i} \hbar \omega_{i} \ll 1$, and weak correlation, $\beta_{i} \hbar \lambda \ll 1$. Here $\lambda$ is the strength of the interaction that created the thermal entangled pair. The maximum efficiency (11) is larger than the Carnot expression when one atom o a pair flies through the cavity $\left(\Delta \bar{n}_{\lambda: 1}>0\right)$ and smaller when the two atoms of a pair pass through it $\left(\Delta \bar{n}_{\lambda: 2}<0\right)$. In both cases, the deviation of the mean occupation number is inversely proportional to the frequency $\omega_{2}$. The total work produced by the engine during one cycle is,

$$
-\langle W\rangle=\frac{1}{\beta_{1}}\left(\frac{\omega_{2}}{\omega_{1}}-1\right)+\left(\frac{1}{\beta_{2}}+\hbar \omega_{2} \Delta \bar{n}_{\lambda}\right)\left(\frac{\omega_{1}}{\omega_{2}}-1\right) .
$$

Assuming that the initial frequency of the oscillator $\omega_{1}$ (as well as $\lambda, \beta_{1}, \beta_{2}$ and the cycle time) are fixed and by optimizing with respect to the second frequency $\omega_{2}$, we find that the power is maximum when $\omega_{1} / \omega_{2}=$ $\sqrt{\beta_{2} /\left(\beta_{1}\left[1+\hbar \beta_{2} \omega_{2} \Delta \bar{n}_{\lambda}\right]\right)}$. As a result, the efficiency at maximum power is given by,

$$
\eta_{\gamma}=1-\sqrt{\frac{\beta_{2}}{\beta_{1}\left(1+\beta_{2}\langle\Delta H\rangle_{C}\right)}}=1-\sqrt{\frac{\beta_{2}^{\mathrm{eff}}}{\beta_{1}}} .
$$

Equation 15 reduces to the Curzon-Ahlborn expression 49] for vanishing correlation and generally exceeds it when one atom flies through the cavity, $\langle\Delta H\rangle_{C}=$ $\hbar \omega_{2} \Delta \bar{n}_{\lambda}>0$. Remarkably, Eq. (15) can be expressed in terms of the same effective temperature as Eq. 111. This remains true for all reservoirs with $\Delta \bar{n}\left(\omega_{2}\right) \sim 1 / \omega_{2}$. A discussion of the efficiency at maximum power for a quantum coherent reservoir [23], for which $\Delta \bar{n}\left(\omega_{2}\right) \sim 1 / \omega_{2}^{2}$, is presented in the Appendix. In the limit of small temperature differences and small $\langle\Delta H\rangle_{C}$, we have $\eta_{\gamma} \simeq$ $\eta_{c} / 2+\beta_{2}^{2}\langle\Delta H\rangle_{C} /\left(2 \beta_{1}\right) \geq \eta_{c} / 2$, when $\langle\Delta H\rangle_{C}>0$; this result therefore lies beyond the range of the usual linear regime 11,13 .

Generalization. The above results can be extended to situations where the two reservoirs are nonthermal. For instance, the efficiency of the quantum Otto engine is,

$$
\eta=1-\frac{\omega_{1}}{\omega_{2}} \frac{\left(\bar{n}_{1}+\Delta \bar{n}_{1}+1 / 2\right)-Q_{2}^{*}\left(\bar{n}_{2}+\Delta \bar{n}_{2}+1 / 2\right)}{\left(\bar{n}_{1}+\Delta \bar{n}_{1}+1 / 2\right) Q_{1}^{*}-\left(\bar{n}_{2}+\Delta \bar{n}_{2}+1 / 2\right)},
$$

where $\Delta \bar{n}_{i}$ is the deviation of the mean occupation number for the nonthermal reservoir $i(i=1,2)$. The positivity of the entropy production for this nonequilibrium configuration leads to the following expression of the hightemperature maximum efficiency,

$$
\eta_{\max }=1-\frac{\beta_{2}^{\mathrm{eff}}}{\beta_{1}^{\mathrm{eff}}}=\eta_{c}+\beta_{2}\left(\frac{\omega_{1}}{\omega_{2}}\langle\Delta H\rangle_{C}-\langle\Delta H\rangle_{A}\right),
$$

with the effective temperatures $T_{2}^{\mathrm{eff}}=T_{2}+\hbar \omega_{2} \Delta \bar{n}_{2} / k=$ $T_{2}+\langle\Delta H\rangle_{C} / k$, as before, and $T_{1}^{\mathrm{eff}}=T_{1}+\hbar \omega_{1} \Delta \bar{n}_{1} / k=$ $T_{2}+\langle\Delta H\rangle_{A} / k$. Here we have defined the deviation, $\langle\Delta H\rangle_{A}=\langle H\rangle_{A}-\langle H\rangle_{A}^{\mathrm{eq}}$, of the energy of the oscillator from its equilibrium value at point $A$. The efficiency (17) is larger than the Carnot efficiency when $\omega_{1}\langle\Delta H\rangle_{C}-\omega_{2}\langle\Delta H\rangle_{A}>0$. It is interesting to note that Eq. 17) only depends on the average deviations at point $A$ and $C$ and not at points $B$ or $D$.

Conclusions. We have used the positivity of the entropy production to compute the maximum efficiency of a quantum heat engine operating between nonthermal stationary reservoirs. We have shown that the latter can be expressed in terms of an effective temperature which characterizes the deviation from equilibrium. We have obtained explicit conditions under which the maximum efficiency exceeds the standard Carnot bound in the presence of either one or two nonthermal reservoirs. We have further derived a generalization of the Clausius statement of the second law on the direction of heat flow between two nonequilibrium systems. Additionally, we have evaluated the efficiency at maximum power of the Otto engine for a quantum correlated reservoir and obtained nonlinear extensions of the Curzon-Ahlborn formula. The efficiency of molecular motors has been experimentally shown to approach unity in some cases [9, 10, far surpassing the efficiency of heat engines and highlighting the advantage of operating away from equilibrium. Our results provide a theoretical framework for a new class of engineered heat engines that interpolate between standard heat engines and molecular motors.

This work was supported by the DFG (contract No LU1382/4-1) and the Alexander von Humboldt Foundation. 


\section{APPENDIX}

A quantum coherent nonthermal reservoir can be created by sending through the optical cavity a beam of thermal three-level atoms whose degenerate ground states are prepared in a coherent superposition with relative phase $\phi$. In the high-temperature limit, the deviation of the mean occupation number is [23,

$$
\Delta \bar{n}_{\phi}=-\frac{1}{\left(\hbar \beta_{2} \omega_{2}\right)^{2}} \varepsilon \cos \phi,
$$

where $\varepsilon$ is proportional to the amplitude of the atomic coherence. Here $\Delta \bar{n}$ is inversely proportional to the square of the frequency $\omega_{2}$. The total work produced by the heat engine can be readily written as,

$$
-\langle W\rangle=\frac{1}{\beta_{1}}\left(\frac{\omega_{2}}{\omega_{1}}-1\right)+\frac{1}{\beta_{2}}\left(\frac{\omega_{1}}{\omega_{2}}-1\right)\left(1-\frac{\varepsilon \cos \phi}{\hbar \beta_{2} \omega_{2}}\right)
$$

By maximizing the power with respect to $\omega_{2}$, keeping all other parameters constant as done in the main text, we find that the power is maximum when the following condition is satisfied,

$$
\frac{\omega_{2}}{\omega_{1}}=\sqrt{\frac{\beta_{2}}{\beta_{1}}}\left[1-\frac{\varepsilon \cos \phi}{2 \hbar \beta_{2} \omega_{1}}\left(1-2 \sqrt{\frac{\beta_{1}}{\beta_{2}}}\right)\right],
$$

in a perturbation expansion for small values of $\varepsilon[?]$. The resulting efficiency at maximum power is then

$$
\eta_{\phi}=1-\sqrt{\frac{\beta_{2}}{\beta_{1}}}\left[1-\frac{\varepsilon \cos \phi}{2 \hbar \beta_{2} \omega_{1}}\left(1-2 \sqrt{\frac{\beta_{1}}{\beta_{2}}}\right)\right] .
$$

The maximum efficiency, $\eta_{\max }=1-\beta_{2}^{\text {eff }} / \beta_{1}$, and the efficiency at maximum power 21) exceed their thermal counterparts when the condition $\cos \phi<0$ is satisfied.

[1] H. B. Callen, Thermodynamics and an Introduction to Thermostatistics (Wiley, New York, 1985).

[2] Y.A. Cengel and M.A. Boles, Thermodynamics. An Engineering Approach, (McGraw-Hill, New York, 2001).

[3] F. Jülicher, A. Ajdari, and J. Prost, Rev. Mod. Phys. 69, 1269 (1997).

[4] P. Reimann, Phys. Rep. 361, 57 (2002).

[5] E.R. Kay, D.A. Leigh, and F. Zerbetto, Angew. Chem. Int. Ed. 46, 72 (2007).

[6] P. Hänggi and F. Marchesoni, Rev. Mod. Phys. 81, 387 (2009).

[7] A. Parmeggiani, F. Jülicher, A. Ajdari, and J. Prost, Phys. Rev. E 60, 2127 (1999).

[8] R. D. Astumian, PNAS 104, 19715 (2007).

[9] S. Toyabe, T. Okamoto, T. Watanabe-Nakayama, H. Taketani, S. Kudo, and E. Muneyuki, Phys. Rev. Lett. 104, 198103 (2010).

[10] S. Toyabe, T. Watanabe-Nakayama, T. Okamoto, S. Kudo, E. Muneyuki, PNAS 108, 17951 (2011).
[11] T. Schmiedl and U. Seifert, Europhys. Lett. 81, 20003 (2008).

[12] M. Esposito, K. Lindenberg, and C. Van den Broeck, Phys. Rev. Lett. 102, 130602 (2009).

[13] M. Esposito, R. Kawai, K. Lindenberg, and C. Van den Broeck, Phys. Rev. Lett. 105, 150603 (2010).

[14] U. Seifert, Phys. Rev. Lett. 106, 020601 (2011).

[15] C. Van den Broeck, N. Kumar, and K. Lindenberg, Phys. Rev. Lett. 108, 210602 (2012).

[16] J.F. Poyatos, J.I. Cirac, and P. Zoller, Phys. Rev. Lett. 77, 4728 (1996).

[17] C.J. Myatt, B.E. King, Q.A. Turchette, C.A. Sackett, D. Kielpinski, W.M. Itano, C. Monroe, and D.J. Wineland, Nature (London) 403, 269 (2000).

[18] S. Pielawa, G. Morigi, D. Vitali, and L. Davidovich, Phys. Rev. Lett. 98, 240401 (2007).

[19] A. Sarlette, J. M. Raimond, M. Brune, and P. Rouchon, Phys. Rev. Lett. 107, 010402 (2011).

[20] S. Diehl, A. Micheli, A. Kantian, B. Kraus, H. P. Büchler, and P. Zoller, Nature Phys. 4, 878 (2008).

[21] F. Verstraete, M.M. Wolf, and J.I. Cirac, Nature Phys. 5, 633 (2009).

[22] Y.-D. Wang and A.A. Clerk, Phys. Rev. Lett. 110, 253601 (2013).

[23] M.O. Scully, M.S. Zubairy, G.S. Agarwal, and H. Walther, Science 299, 862 (2003).

[24] R. Dillenschneider and E. Lutz, Europhys. Lett. 88, 50003 (2009).

[25] M. O. Scully, Phys. Rev. Lett. 88, 050602 (2002).

[26] B. Lin and J. Chen, Phys. Rev. E 67, 046105 (2003).

[27] Y. Rezek and R. Kosloff, New J. Phys. 8, 83 (2006).

[28] H.T. Quan, Y.X. Liu, C.P. Sun, and F. Nori, Phys. Rev. E 76, 031105 (2007).

[29] O. Abah, J. Rossnagel, G. Jabob, S. Deffner, F. SchmidtKaler, K. Singer, and E. Lutz, Phys. Rev. Lett. 109, 203006 (2012).

[30] M. Campisi, P. Talkner, and P. Hänggi, Phys. Rev. Lett. 102, 210401 (2009).

[31] S. Hilt, B. Thomas, and E. Lutz, Phys. Rev. E 84, 031110 (2011).

[32] T. Hill, Introduction to Statistical Thermodynamics, (Adison-Wesley, Reading, 1960), Chap. 17.

[33] C. Jarzynski, Annu. Rev. Condens.Matter Phys. 2, 329 (2011).

[34] For any nonnormalized nonequilibrium operator $\rho_{2}^{\prime}$, we define the normalized operator $\rho_{2}=\rho_{2}^{\prime} / \operatorname{tr}\left\{\rho_{2}^{\prime}\right\}$. We then have $\Delta H_{2}=-\left[\ln \rho_{2}+\ln \operatorname{tr}\left\{\rho_{2}^{\prime}\right\}\right] / \beta_{2}-H_{2}$.

[35] A nonzero $\Delta H_{2}$ may lead to nonthermally distributed population and/or nondiagonal energy matrix elements of $\rho_{2}$, as in the example of the coherent reservoir 23 .

[36] E. Fermi, Thermodynamics, (Dover, New York, 1956).

[37] K. Husimi, Prog. Theor. Phys. 9, 381 (1953).

[38] S. Deffner and E. Lutz, Phys. Rev. E 77, 021128 (2008).

[39] S. Deffner, O. Abah, and E. Lutz, Chem. Phys. 375, 200 (2010).

[40] R. Alicki, J. Phys. A 12, L103 (1979).

[41] M.A. Nielsen and I.L. Chuang, Quantum Computation and Quantum Information, (Cambridge, Cambridge, 2000).

[42] S. Deffner and E. Lutz, Phys. Rev. Lett. 107, 140404 (2011).

[43] The derivation can be easily extended to two nonequilibrium systems: the two temperatures will be replaced by the corresponding effective temperatures. 
[44] R. Clausius, The Mechanical Theory of Heat, (Macmillan, London, 1879), page 78.

[45] R.C. Tolman, The Principles of Statistical Mechanics, (Oxford, Oxford, 1938), Sect. 127.

[46] M.H. Partovi, Phys. Lett. A 137, 440 (1989).

[47] In a similar way, the evaluation of the efficiency of a car engine does not include the energetic cost of extracting, refining and transporting oil to the gas station.

[48] J. Parrondo and B. de Cisneros, Appl. Phys. A: Mater. Sci. Process. 75, 179 (2002).

[49] F.L. Curzon and B. Ahlborn, Am. J. Phys. 43, 22 (1975). 